\title{
The Association of Clinical Pathologists: 99th spring meeting
}

The 99th spring meeting was held at the Holiday Inn, Plymouth on 20-22 April 1977. Abstracts of scientific communi cations follow.

\author{
Obliterative bronchiolitis and rheumatoid \\ disease
}

BRYAN CORRIN, DUNCAN M. GEDDES, MARGARET TURNER-WARWICK, DERRICK A. BREWERTON (St. Thomas', Brompton and Westminster Hospitals, London) In childhood, viral infection of the lung commonly causes a bronchiolitis with airways obstruction. Occasionally this is followed by an obliterative bronchiolitis which may prove fatal. In adults, obliterative bronchiolitis has rarely been reported except after inhalation of chemical fumes. During 1970-76 six patients without previous chest disease were admitted with progressive respiratory failure due to airways obstruction. Five have died and four were examined post mortem when an obliterative bronchiolitis was identified. Classical, seropositive rheumatoid arthritis was present in five of the six patients while the sixth had circulating antinuclear antibodies. The respiratory illness was characterised in each case by a relatively rapid onset of breathlessness, progressing over a few months to complete incapacity or death. Loud crackles and a short midinspiratory squeak were heard throughout the lungs. All chest radiographs were essentially normal. Lung function tests showed a reduction in spirometric volumes with airflow obstruction and hyperinflation. In view of the apparent rarity of bronchiolitis in adults, the finding of this condition in several patients with rheumatoid arthritis suggests that there may be an association. The condition is difficult to diagnose even after death and may occur more frequently than has been appreciated. The postmortem findings are described.

The prevalence of yellow bodies in lymph nodes at different sites

A. J. C. TUDWAY (Medical School, Univer- sity of Bristol) Yellow bodies have been reported in lymph nodes of sarcoid patients (Wesenberg, 1966) and in other diseases (Boyd and Valentine, 1970).

Their prevalence in eight lymph node groups has been studied in postmortem material from 46 individuals chosen at random. One lymph node was sought from each group in every individual and a total of 359 were collected. In addition, 170 cystic lymph nodes from a consecutive series of cholecystectomy specimens were examined. One haematoxylin and eosin stained paraffin section of each lymph node was searched for the bodies which appear as round or oval structures, 1-10 $\mathrm{m} \mu$ diameter, usually lying within sinuses.

Yellow bodies were found in $82(22.8 \%)$ of the postmortem nodes. They were present in $19.6 \%$ axillary, $13.0 \%$ inguinal, $11.1 \%$ paratracheal, $22.2 \%$ lesser curve, $28.3 \%$ porta hepatis, $27.9 \%$ cystic, $35.6 \%$ mesenteric, and $25.6 \%$ para-aortic lymph nodes. Of the surgical series of cystic nodes, $52(30.6 \%)$ contained bodies.

The presence of bodies did not relate significantly to age or sex. They were present in two otherwise healthy individuals who died of trauma. There was no significant difference between the postmortem and surgically excised cystic nodes, though the latter were all associated with cholecystitis. The five abdominal lymph node groups taken together showed a greater prevalence of bodies than the superficial nodes (axillary and inguinal groups together) which is just significant $(P<$ $0.05)$.

Yellow bodies appear to be common in several sites. Their predilection for abdominal nodes may be relevant to their suggested ceroid nature (Doyle et al., 1973; Sieracki and Fisher, 1973) and the high lipid content of many of these nodes.

\section{References}

Boyd, J. F., and Valentine, J. C. (1970). J. 784
Pathol., 102, 58-60.

Doyle, W. F., et al. (1973). Arch. Pathol., 96, 320-326.

Sieracki, J. C., and Fisher, E. A. (1973) Amer. J. clin. Pathol., 59, 248-253.

Wesenberg, W. (1966). Arch. klin. exp‡ Dermatol., 227, 101-107.

Realistic in vitro tests of antibiotic activit

M. BAKHTIAR AND S. SELWYN (Departmen $\frac{\bar{\varphi}}{4}$ of Bacterioiogy, Westminster Medicat School, London) ' Although generally performed with complete confidence, the. conventional in vitro tests for antibiotig sensitivity are largely unrealistic and the results obtained are often of doubtfuf validity in vivo. The situation is particu larly unsatisfactory in relation to beta lactam antibiotics (Selwyn, 1976). The first major problem is that routine soli and liquid culture media bear no relation 3 ship either to plasma or extracellular fluich through which antibiotics are distributed in. the body, or to the tissues where most infections occur. Thus, the addition of appropriate amounts of human serun albumin to test media considerably. increases the conventionally derived miniô mum inhibitory concentration (MIC) o色 the majority of antibiotics and has an evero more dramatic effect on minimum bacteria concentration (MBC) values.

The second important problem in con ventional tests is the manner in which of freshly inoculated micro-organism imme diately encounters a relatively high cone centration of antibiotic before growth is established or products such as betag lactamases have reached active concen trations. This type of encounter resemble the prophylactic use of antibiotics but now their therapeutic administration.

Pre-incubation of inoculated culture media before the addition of antibiotic㞧 produces striking decreases in the apparen? sensitivity of organisms to many drugs Only part of this effect is due to increased 
inoculum size, and the effect is often enhanced if subinhibitory levels of drugs are pre-diffused also.

The final major problem is to determine a true end-point for the MIC of an antibiotic. The values obtained in routine dilution tests are inaccurate partly due to the discontinuity of the concentration series and partly because of a complex and changing pattern of inhibition in dilutions beyond the conventional MIC, particularly during the first half of the growth cycle (Greenwood, 1976). In contrast, MBCs derived from these procedures under defined conditions are found to be more consistent than MICs. Other methods of estimating MICs, such as turbidometric and enzyme inhibition procedures-and their modifications used in assaying drugs -have been evaluated and compared with the standard culture methods by means of accurate viable and total cell counts. Striking inconsistencies in the results have been revealed; these possess diverse but reproducible characteristics for different antibiotics. Routine in vitro tests require modifications in the three areas discussed before the results can be confidently extrapolated to antibiotic therapy in man.

\section{References}

Greenwood, D. (1976). Differentiation of mechanisms responsible for inoculum effects in the response of Escherichia coli to a variety of antibiotics. J. antimicrobial Chemotherapy, 2, 87-95.

Selwyn, S. (1976). Rational choice of penicillins and cephalosporins. Lancet, 2 , 616-19.

The in vivo behaviour of salmonellas in the intestinal tract of the chick: an animal model of pathological and public health importance

P. C. B. TURNBULL AND JOAN E. RICHMOND (Food Hygiene and Virus Reference Laboratories, Central Public Health Laboratory, Colindale Avenue, London NW9 $5 H T$ ) A previous study on the pathogenesis of salmonella in the chicken (Turnbull and Snoeyenbos, 1974; Avian Dis., 18:153) indicated that the day-old chick would provide an excellent model for detailed study of salmonella enteritis.

Results of conventional histology together with fluorescent antibody and ultrastructural examination of ligated caeca in day-old chicks 5 to 7 hours after inoculation with $S$. enteritidis are now presented. Entry of the organism appeared to be more an 'uptake' than an 'invasion' and to be associated with distinct evaginations from the epithelial surface. In the epithelial cells, salmonellas were confined within membrane-bound vacuoles, and epithelial damage resulted only from uptake of very large numbers of the organisms. The vacuoles moved towards the lamina propria, their contents often becoming increasingly osmiophilic.

Occasional organisms were found in the lamina propria both free and intracellularly, and it is proposed that the lamina propria vessels supply the route to bacteraemia. By light microscopy alone, microcolony development within the epithelium and lamina propria was apparent at 12 hours.

Also by light microscopy, similar invasion was seen in ligated duodenum, midgut, and caudal ileum, demonstrating that entry may not necessarily be confined to one intestinal region.

\section{Flucloxacillin in bone}

P. F. UNSWORTH, F. W. HEATLEY, I. PHILLIPS (St Thomas' Hospital Medical School, London SE1 7EH) Ten patients undergoing total hip replacement for osteoarthritis were each given intramuscular flucloxacillin before operation, and bone and serum were removed simultaneously at operation. Trabecular and compact bone was partly dried and then reduced to powder, before antibiotic was extracted with buffer. The concentration of flucloxacillin in bone washings and serum was determined by well diffusion assay. The mean levels of flucloxacillin detected were: in serum $7.8 \mathrm{mg} / 1$, in trabecular bone 1.3 $\mathrm{mg} / \mathrm{l}$, and in compact bone $0.9 \mathrm{mg} / 1$. The significance of these findings is discussed with reference to the prophylactic use of flucloxacillin in hip replacement.

Measurements were made of the amount of blood present in washings of ground bone, which were estimated to account for no more than about $25 \%$ of the flucloxacillin present in bone.

\section{Chlorhexidine-detergent and other alter- natives to hexachlorophane for use in maternity nurseries}

ESTHER FARES, S. SELWYN, TUSHNA SETHNA (Department of Bacteriology, Westminster Medical School, London) Bacterial colonisation of the skin of newborn infants has been studied for two and a half years, using an efficient semiquantitative swabbing technique (Seliwyn and Ellis, 1972). Two identical maternity nurseries with the $\overrightarrow{\vec{F}}$ same staff provided the ideal setting for comparative trials of different bathing pro- $\frac{O}{-}$ cedures. The main purpose of this part of $\frac{\overline{\bar{D}}}{\bar{D}}$ the investigation was to evaluate alter- $\frac{\bar{D}}{\vec{D}}$ natives to hexachlorophane, in view of $\underset{\mathcal{D}}{\mathscr{Q}}$ recent concern about the potential toxicity of this antiseptic (Kensit, 1975).

Five bathing regimes were allocated to $\overrightarrow{0}$ eight groups, each containing 60 infants, $\overrightarrow{\vec{H}}$ in the following sequence. During the first $\vec{\omega}$ phase of the study, babies in one nursery were bathed using simple soap, and? unmedicated dusting powder was applied whenever necessary; in the other nursery, $\infty$ hexachlorophane was incorporated in the same soap and powder bases. During the $\stackrel{\infty}{\oplus}$ second phase, an unmedicated buffered $O$ solution (Infacare) was added to the bath water in one nursery, and inert powder was also applied; the second nursery again used the hexachlorophane regime. क In the third phase, Infacare was compared $\stackrel{\oplus}{+}$ with a chlorhexidine-detergent solution $\vec{\theta}$ (Hibiscrub) used at a concentration of $1 \%$ ㄱ in the bath water. During the final phase, Hibiscrub was used as a $10 \%$ strength for soaping the babies at bath-time in one nursery, and simple soap was used in the other nursery. Throughout the study, daily swabs were taken from the anterior nares, $\stackrel{\mathbb{D}}{\triangle}$ axillae, and umbilicus.

Colonisation with Staphylococcus aureus at all sites was consistently lowest in the hexachlorophane groups, but the simultaneous reduction in commensal bacteria may explain the tendency for overgrowth by Gram-negative bacilli in these groups. : Infacare bathing was convenient and 3 inexpensive, and was associated with a relatively low incidence of carriage and $\frac{0}{3}$ persistence of Staph. aureus on discharge from hospital, even though transient ? colonisation was not reduced. This $D$ satisfactory effect could have been due to the maintenance of the skin's protective 'acid mantle'.

Although a $1 \%$ Hibiscrub bath proved $N$ less efficient than hexachlorophane, better $N$ results were obtained by soaping with $10 \% \mathrm{~W}$ Hibiscrub. The preparation was found to be highly acceptable and, in contrast to hexachlorophane, showed little tendency $\mathbb{D}$ to select Gram-negative bacilli.

\section{References}

Kensit, J. G. (1975). Hexachlorophane: toxicity and effectiveness in prevention of 
sepsis in neonatal units. J. antimicrob. Chemother., 1, 263-72.

Selwyn, S., and Ellis, H. (1972). Skin bacteria and skin disinfection reconsidered. Brit. med. J., 1, 136-40.

Serum enzymes as tumour markers in the diagnosis of hepatic metastatic carcinoma

P. R. BECK, A. BELFIELD, L. H. BLUMGART, R. J. SPOONER, C. B. WOOD (Departments of Biochemistry and Surgery, Royal Infirmary, Glasgow) It is aimed to present an analysis of the results obtained on approximately 400 patients with local and metastatic colonic carcinoma utilising six serum enzymes, and to compare these serum enzymes with other methods for the diagnosis of metastatic carcinoma.

Scanning electron microscopy in the cutaneous inflammatory response

G. HUDSON, R. J. SOKOL, T. E. DURRANT, E. K. BLACKBURN (Royal Infirmary, Sheffield)
The skin window technique has been adapted to allow cells of the cutaneous inflammatory response to be visualised by scanning electron microscopy (Sokol, Durrant, and Hudson, J. clin. Path., 29, $760,1976)$. These methods have been used to study cells of the normal response in 22 subjects at 24 and 48 hours.

With slow-dried preparations, the cells appeared flattened and the nuclear outline was usually evident. Granulocytes differed from macrophages by having significantly smaller measurements for whole cell area and cell diameter but larger values for nuclear :cytoplasmic ratio.

With critical-point dried specimens there was usually a wealth of surface detail and no evidence of underlying nucleus or organelles. Typical granulocytes were up to $10 \mu \mathrm{m}$ in diameter, had delicate curved surface folds, and variable numbers of fine filopodia extending outwards on the coverslip from the cell periphery. Typical macrophages were larger, their surface usually being covered with tightly packed knob-like microvilli (although smooth areas were sometimes seen) and the periphery showing many filopodia and occasional broader projections. Son macrophage filopodia took origin from the free surface of the cell but ran to te attached to the coverslip for the greatef part of their extent, or became "continuouss with a filopodium of another cell. Som cells could not be classified. Preliminary observations suggest that changes in these appearances may be found in disease states.

Tumours of the nose and sinuses

INGLE WRIGHT (ENT Pathology, Universitú of Manchester) The tumour materiab from the nose and sinuses in the Manches ter Royal Infirmary surgical histolog $\not$ index 1954-76 is reviewed with particula interest in (i) the transitional ('inverted' papilloma, which appears to deserve a bet ter prognosis than has been accorded to $\mathbb{B}$ in the past; and (ii) squamous carcinom年 of the antrum, a 'silent' area with correspondingly poor prognosis. 